\title{
Magnetic Resonance Imaging Brain and Electroencephalogram (EEG) in the Evaluation of New-Onset Seizures in a Tertiary Care Centre of Nepal
}

\author{
Pokharel BR ${ }^{1}$, Upadhaya $\mathrm{P}^{2}$, Sharma GR ${ }^{3}$, Budathoki SJ ${ }^{4}$, Maharjan AMS ${ }^{5}$, Kharel G ${ }^{6}$, Phuyal $\mathrm{S}^{7}$. \\ ${ }^{1}$ Principal Consultant Neurologist - Nepal Mediciti Hospital \\ ${ }^{2}$ Registrar Department of Neurology- Nepal Mediciti hospital \\ ${ }^{3}$ Principal Consultant Neurosurgeon - Nepal Mediciti Hospital \\ ${ }^{4}$ Medical officer Department of Neurology - Nepal Mediciti hospital \\ ${ }^{5}$ Lecturer Consultant Neurologist - Upendra Devkota Memorial National Institute of Neurological and Allied Sciences, Kathmandu \\ ${ }^{6}$ Assistant Prof - Department of Neurology - Upendra Devkota Memorial National Institute of Neurological and Allied Sciences, \\ Kathmandu, \\ ${ }^{7}$ Consultant Interventional Neuroradiologist - Upendra Devkota Memorial National Institute of Neurological and Allied Sciences, \\ Kathmandu,
}

Keywords: epilepsy; electroencephalography; magnetic resonance imaging; seizure

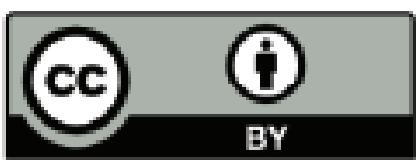

This work is licensed under a Creative Commons Attribution 4.0 Unported License.

\begin{abstract}
Introduction: Seizure is a common neurological condition with multiple etiological factors. This study aims to evaluate the role of magnetic resonance imaging (MRI) Brain and electroencephalography (EEG) in the diagnosis of newonset seizures in the Nepalese population.

Methods: A total of 106 patients aged between 7 to 85 years of age with first onset seizure, who underwent MRI and EEG were enrolled in the study.

The sensitivity of MRI and EEG for the diagnosis of seizure when used in combination was compared with that of MRI or EEG alone.

Results: Out of 106 patients, $58.5 \%(\mathrm{n}=62)$ were males and $41.5 \%(n=44)$ were females. In $52.8 \%(n=56)$ of the patients, there was epileptogenic lesion in MRI, and $39.6 \%(n=42)$ of the patients had an abnormal EEG. The combination of MRI with EEG was significantly better than either MRI or EEG alone in the diagnosis of seizures $(\mathrm{p}<0.001)$.

Conclusion: MRI and EEG are frequently used for the evaluation of seizures. MRI Brain when used in combination with EEG significantly improves the diagnostic accuracy of seizures.
\end{abstract}

\section{Introduction}

Seizure is a paroxysmal alteration of the neurologic function caused by an excessive, hyper synchronous discharge of the neurons in the brain resulting in acute changes in the clinical state of the person. Seizure is the common manifestation of several disease conditions, such as central nervous system infections, biochemical abnormalities, genetic conditions, adverse drug effects, and intracranial mass lesions.1 Epilepsy is a disorder characterized by two or more unprovoked seizures occurring at least 24 hours apart, or one unprovoked seizure and a possibility of further seizures of at least $60 \%$ after two unprovoked seizures, occurring over the next 10 years. 2

\footnotetext{
*Corresponding Author:

Dr Babu Ram Pokharel

Principal Consultant Neurologist and Head Department of Neurology

Nepal Mediciti Hospital

E mail: drbrpokh@gmail.com ORCID: 0000-0002-5577-9643

Ph. No; 9841929745
} 
The prevalence of epilepsy in Nepal is approximately 7.3 per 1000 population. 3 Nepal has a huge geographical diversity and the majority of the people, especially those living in rural areas, have limited access to healthcare facilities. Neurocysticercosis with calcified lesions is the most common cause for epilepsy in Nepal, which accounts for almost $17 \%$ of all the cases in the country. 4

MRI of brain with dedicated epilepsy sequences is the investigation of choice in patients with epilepsy. 5 In recent years, there has been a significant improvement in neuroimaging, its post-processing techniques, and the availability of trained staff. The identification of structural and functional causes of epilepsy has become more accurate.6 EEG is a non-invasive, cost-effective, and bedside procedure that has an established role in the diagnosis, classification, and management of epilepsy.7, 8 The purpose of this study was to compare the effectiveness of MRI and EEG in the diagnosis of first-onset seizures, when used alone and when used in combination,.

\section{Methods}

It is a retrospective study performed in all patients with first-onset seizure presenting to Neurology departments of Nepal Mediciti Hospital, Lalitpur from June 2019 to May 2021. MRI and EEG were performed after the seizure episodes. The patients were classified into different age groups child, adolescents, adults and seniors adults (WHO classification). The imaging of the patient was further sub-grouped into structural and non-structural causes. The seizure was defined relative to the symptoms and signs of the patient and was classified using the International League Against Epilepsy (ILEA) classification.9 The sample size was estimated assuming the proportion of individuals with MRI positivity of $80 \%$, with absolute precision of $10 \%$ and desired confidence interval of $95 \%$. The required sample size was determined to be 96 , using the formula n' $=\mathrm{t}^{2} \times \mathrm{p}(1-\mathrm{p}) / \mathrm{m}^{2}$ where, $\mathrm{n}=$ required sample size, $\mathrm{t}=$ confidence interval at $95 \%$ (standard value 1.96), $\mathrm{p}=$ estimated prevalence of positive result, and $\mathrm{m}=$ estimated precision. Patients with non-epileptic seizure, known seizure disorder, and contraindication to performing MRI were excluded from the study.

The patients' data was entered into Microsoft Excel sheet (Microsoft Office 2019, USA) and analysed using SPSS version 20 (IBM SPSS, USA). Descriptive statistical analysis was done and if required, the data were tabulated with appropriate means. The sensitivity and specificity of the test were calculated separately as well as in combination. All the qualitative variables were expressed in terms of proportion. Chi-square test of significance $(\mathrm{p}<0.05)$ was used to test for the difference in proportion. The correlation between MRI brain and EEG were studied.

\section{Result}

A total of 106 patients with new-onset seizure were included in this study. The patients were either admitted to the hospital or had visited the out-patient's department during the study period. The mean age of the patients was $31.97 \pm 15.44$ years. Among them, $58.5 \%(n=62)$ and $41.5 \%(n=44)$ were males and females respectively. The majority of the patient belonged to the adult population as shown in Figure 1.
Figure 1: Age distribution of the patients

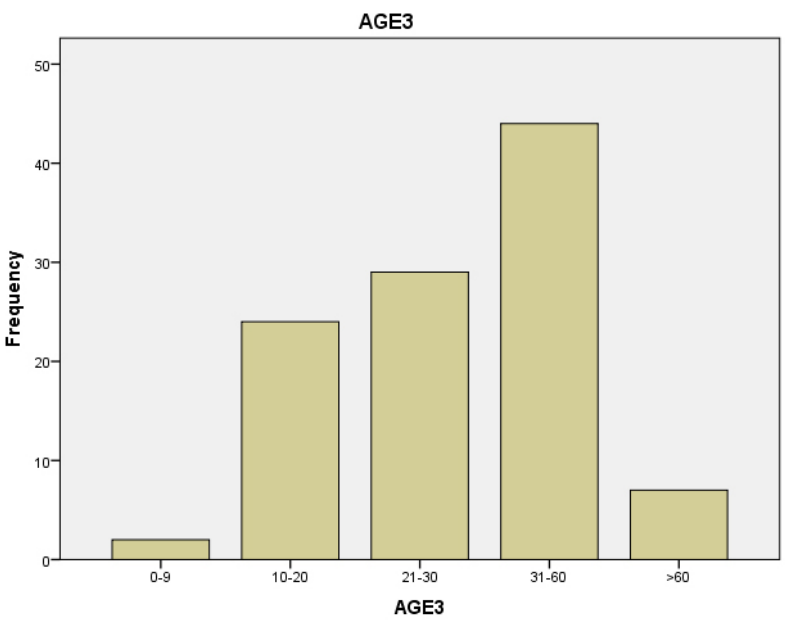

The majority of the patients had a generalized tonic-clonic seizure (GTCS) (Figure 2).

Figure 2: The frequency of different types of seizure

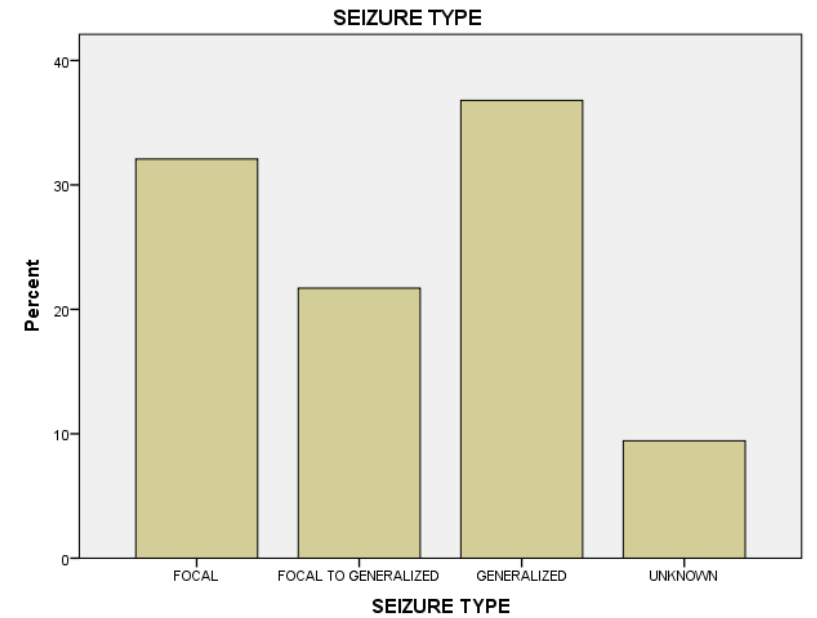

Table 1: MRI and EEG reporting in epilepsy patient

\begin{tabular}{|c|c|c|}
\hline $\begin{array}{c}\text { Monitoring } \\
\text { Modality }\end{array}$ & & Frequency (\%) \\
\hline \multirow{2}{*}{ MRI findings } & Abnormal & $56(52.8)$ \\
\cline { 2 - 3 } & Normal & $50(47.2)$ \\
\hline \multirow{2}{*}{ EEG findings } & Abnormal & $42(39.6)$ \\
\cline { 2 - 3 } & Normal & $64(60.4)$ \\
\hline
\end{tabular}

Table 2: Sensitivity of MRI and EEG

\begin{tabular}{|l|c|c|l|}
\hline & Positive & Negative & $\begin{array}{l}\text { Sensitivity (95\% } \\
\text { Confidence Interval) }\end{array}$ \\
\hline MRI & 56 & 50 & $52.83 \%(0.429-0.626)$ \\
\hline EEG & 42 & 64 & $39.6 \%(0.303-0.496)$ \\
\hline MRI + EEG & 75 & 31 & $70.75 \%(0.611-0.792)$ \\
\hline
\end{tabular}


Magnetic Resonance Imaging Brain and Electroencephalogram (EEG) in the Evaluation of New-Onset Seizures in a Tertiary Care Centre of Nepal

\begin{tabular}{|c|c|c|c|c|}
\hline MRI & \multicolumn{2}{|c|}{ EEG } & Total & P-value \\
\hline & Abnormal & Normal & & \\
\hline Abnormal & $23(54.8 \%)$ & $33(51.6 \%)$ & 56 & 0.071 \\
\hline Normal & $19(38 \%)$ & $31(62 \%)$ & 50 & \\
\hline Total & $42(39.6 \%)$ & $64(60.3 \%)$ & 106 & \\
\hline
\end{tabular}

Table 3: Comparison of MRI and EEG

Table 4: Comparison of MRI and EEG combined with EEG alone

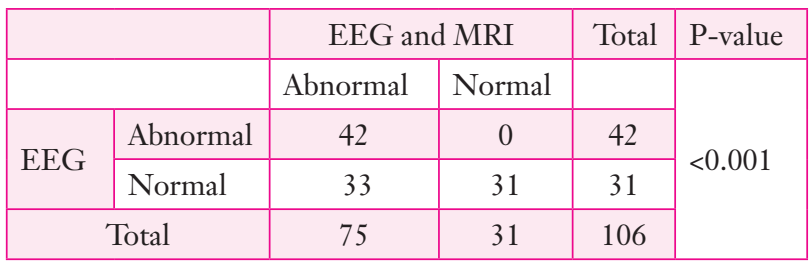

Table 5: Comparison of MRI and EEG combined with MRI alone

\begin{tabular}{|c|l|c|c|c|c|}
\hline \multicolumn{2}{|c|}{} & \multicolumn{2}{c|}{ EEG and MRI } & Total & P-value \\
\hline \multirow{2}{*}{ EEG } & Abnormal & Normal & & \\
\cline { 2 - 5 } & Abnormal & 56 & 0 & 56 & \multirow{3}{*}{$<0.001$} \\
\cline { 2 - 5 } & Normal & 19 & 31 & 31 & \\
\hline \multicolumn{2}{|c|}{ Total } & 75 & 31 & 106 & \\
\hline
\end{tabular}

\section{Discussion:}

About $5 \%$ of the general population will experience one unprovoked seizure during their lifetime 10, less than half of these will develop epilepsy 11 . Various investigations are available for detection of the structural and the functional abnormalities of a seizure which include EEG, cerebral structural imaging (MRI and magnetic resonance spectroscopy), magnetoencephalography (MEG) and cerebral function imaging (SPECT and PET). The limitations of each test in localizing the seizure focus must be recognized. Not all tests will show localizing abnormalities and the foci determined by different tests may be unrelated. The clinical history of the patient is extremely important in the evaluation of a patient with seizure and epilepsy. In out-ofhospital seizures, a video-recording of the episode of the seizure, if available, is helpful to make a clinical diagnosis. Seizures are broadly classified as focal, focal with generalized, or unknown. The common causes of seizures are different in patients of different age groups. 12

In our study, a total of 106 patients underwent MRI of the brain for evaluation of seizures, among whom $56(52.83 \%)$ patients had imaging findings like infarction, neurocysticercosis, calcified granuloma, or tumor. MRI provides optimal information for finding out temporal lobe (mesial temporal lobe) and frontal lobe lesions, which are the usual cause of the focal seizure with impaired awareness. MRI provides additional information on structural lesions, like primary or metastatic tumour, infection, inflammation, acute infarct or hemorrhage.13 MRI is an important step in the evaluation of seizure; however, in some patients, CT scan of the head may provide rapid radiological diagnosis.

The patients in the study were subjected to EEG examination. If the patients did not have any contraindication, they were subjected to the EEG lab for at least $30 \mathrm{~min}$ and provocation factors like hyperventilation and photosensitivity were tested in each case. EEG is one of the important tools in the diagnosing and analysis of seizures. It represents the electrical activity produced by perpendicular neurons to the brain surface. For the detection of seizure, neurons in at least an area of 6 square $\mathrm{cm}$ need to be electrically hyperactive. Internationally recognized system for EEG is the 10-20 system; the 10- 20 system refers the distance of electrode placements in the scalp from each other 10 $\%$ or $20 \%$ forth back and right-left distance.14.15

Interictal EEG is a safe, cheap and widely available tool for evaluation of the seizure. With the advent of modern neuroimaging and prolonged video-EEG monitoring, interictal EEG recording remains an important in diagnosis and prognostication of patient with seizure. EEG abnormalities have been consistently associated with a higher risk of patients had recurrence $16-19$

In our study, $40 \%$ of the EEG abnormalities. The abnormal al EEG refers to the presence of spikes or spikes with slow waves. The presence of spikes or spikes with slow waves predicts the seizure. The EEG also helps to localize ad classify the type of seizure as either localized or generalized. In patients with firstonset seizures, Gilbert et al showed a wide variation of EEG findings in the detection of seizures. 20

A large portion of patients has no EEG changes or structural abnormalities. In patients, without abnormalities on EEG or neuroimaging, incorporating both EEG and MRI brain together can help in diagnosis and also to localized seizure focus helping surgical treatment in drug resistant cases. MRI and EEG are important modalities in the investigation of seizures. They can be used alone or in combination for the evaluation of seizures. In this study, all the patients had undergone at least $30 \mathrm{~min}$ of EEG and MRI of the brain. The combination of MRI and EEG provided significantly better diagnostic yield compared with either modality when used alone. Similar results were found in a study done by Mohan B et al. 21

The presence of an MRI abnormality concordant with the ictal onset in EEG is independently predictive of excellent postsurgical seizure control in such patient. 22

This study reinforces the need to upgrade the services of MRI and EEG in other parts of the country in the evaluation of seizures.

\section{Conclusion}

The limitation of the study is that it is a single-center study. Seizure and epilepsy are common neurological conditions. There are different causes of seizures in patients of different age groups. Magnetic resonance imaging is important to find out different the structural causes associated with seizures. However, when MRI and EEG are used together, the detection of seizure activity is improved compared with when either modality is used in isolation.

\section{References}

1. Manford, M. Recent advances in epilepsy. J Neurol 2017: 264, 1811-1824.

2. Thijs RD, Surges R, O'Brien TJ, et al. Epilepsy in adults. Lancet. 2019: 689-701

3. Kafle, D. Oli, K. Clinical Profile of Patients with Recurrent Seizure in Tertiary Care Hospital in Nepal. Kathmandu University Medical Journal, 2015: 12(3), 202-206. 
4. Rao KS, Adhikari S, Gauchan E, et al. Time trend of neurocysticercosis in children with seizures in a tertiary hospital of western Nepal. PLOS Neglected Tropical Diseases 2017 :11(5)

5. Scott N. Atlas. Magnetic Resonance Imaging of the brain and spine. 4th edition: 2008; 307-339.

6, Sidhu MK, Duncan JS, Sander JW. Neuroimaging in epilepsy. Curr Opin Neurol. 2018; 31(4):371-378

7. Leach JP, Stephen LJ, Selveta c et al. . Which el ectroencephalography (EEG) for epilepsy? The relative usefulness of different EEG protocols in patients with possible epilepsy. Journal of neurology, neurosurgery, and psychiatry. 2006: 77. 1040-2.

1. Sierra-Marcos A, Bermejo PE, Manso Calderón R et al Lacosamide for epileptic seizures in patients with comorbidities and unusual presentations of epilepsy. CNS Drugs. $2011: 5 ;: 17-26$

2. Hesdorffer DC, Logroscino G, Benn EK et al. Estimating risk for developing epilepsy: a population-based study in Rochester, Minnesota. Neurology. 2011; 76:23-27.

3. Hauser WA, Annegers JF, Kurland LT. Incidence of epilepsy and unprovoked seizures in Rochester, Minnesota: 1935_1984. Epilepsia 1993; 34: 453-468

11. Berg AT, Shinnar S. The risk of seizure recurrence following a first unprovoked seizure: a quantitative review. Neurology 1991; 41: 965-972.

12. Scheffer IE, Berkovic S, Capovilla G, et al ILAE classification of the epilepsies: Position paper of the ILAE Commission for Classification and Terminology. Epilepsia. 2017: 58(4):512-521

13. William G. Bradley and Randolph B. Shey, MR Imaging Evaluation of Seizures Radiology 2000 214:3, 651-656 .

14. E. D. Übeyli,, Statistics over features: EEG signals analysis, Computers in Biology and Medicine, 2009:. 39. 733-741.

15. S. Sanei and J. A. Chambers, EEG Signal Processing, Centre of Digital Signal Processing, Cardiff University, Cardiff, UK, 2007

16. Berg AT, Shinnar S. The risk of seizure recurrence following a first unprovoked seizure: a quantitative review. Neurology 1991; 41: 965-972.

17. Camfield PR, Camfield CS, Dooley JM, et al.. Epilepsy after a first unprovoked seizure in childhood. Neurology 1985; 35: 1657-1660.

18. Stroink H, Brouwer OF, Arts WF et al The first unprovoked, untreated seizure in childhood: a hospital based study of the accuracy of the diagnosis, rate of recurrence, and long term outcome after recurrence. Dutch study of epilepsy in childhood. J Neurol Neurosurg Psychiatry 1998; 64: 595600 .

19. Hirtz D, Ashwal S, Berg A, et al. Practice parameter: Evaluating a first nonfebrile seizure in children: report of the Quality Standards Subcommittee of the American Academy of Neurology, the Child Neurology Society, and the American Epilepsy Society. Neurology 2000; 55: 616623.
20. Gilbert DL, Sethuraman G, Kotagal U, et al . Meta-analysis of EEG test performance shows wide variation among studies. Neurology 2003; 60(3):564.

21. Mohan B, Ranoji. M, Karthik G A, Vimal R, Utility of magnetic resonance imaging (MRI) and it's comparison with electroencephalogram (EEG) in First onset seizures in children. International Journal of Contemporary Medicine Surgery and Radiology. 2018;3(2):B4-B7

22. Radahkrishnan K, So E, Silbert P, et al. Predictors of outcome of anterior temporal lobectomy for intractable epilepsy. A multivariate study. Neurology 1998; 51:465-71 